\title{
Cuidando da Família de Pacientes em Situação de Terminalidade Internados na Unidade de Terapia Intensiva*
}

\section{Caring for the Families of Terminally ill Patients in the Intensive Care Unit}

\author{
Márcio Soares, $P h D^{\dagger}$
}

\section{RESUMO}

JUSTIFICATIVA E OBJETIVOS: O cuidado dos familiares é uma das partes mais importantes do cuidado global dos pacientes internados nas unidades de terapia intensiva (UTI). No contexto de um paciente terminal ou no qual as perspectivas de recuperação são muito improváveis, esta face do cuidado assume uma importância ainda maior, pois na maioria das vezes o paciente não estará desperto, e será preciso lidar e cuidar dos seus familiares. Os familiares têm necessidades específicas e apresentam freqüências elevadas de estresse, distúrbios do humor e ansiedade durante o acompanhamento da internação na UTI, e que muitas vezes persistem após a morte do seu ente querido.

CONTEÚDO: Foram selecionados artigos sobre o cuidado de familiares de pacientes em situação de terminalidade na UTI publicados nos últimos 20 anos na PubMed.

CONCLUSÕES: A literatura recente está repleta de evidências de que estratégias voltadas para os familiares como a melhoria da comunicação, da prevenção de conflitos e do conforto espiritual, para citar algu-

1. Médico do Centro de Tratamento Intensivo, Instituto Nacional de Câncer, Rio de Janeiro; Mestre e Doutor em Clínica Médica pela UFRJ, Título de Especialista em Medicina Intensiva pela AMIB; Membro do Comitê Coordenador da Brazilian Research in Intensive Care Network - BRICNet

Apresentado em 05 de novembro de 2007

Aceito para publicação em 10 de dezembro de 2007.

Endereço para Correspondência:

Dr. Márcio Soares

Núcleo de Pesquisa Clínica em Medicina Intensiva

Instituto Nacional de Câncer - INCA

Centro de Tratamento Intensivo - $10^{\circ}$ Andar

Praça Cruz Vermelha, 23

20230-130 Rio de Janeiro, RJ

Fone: (21) 2506-6120; Fax: (21) 2294-8620

E-mail: marciosoaresms@yahoo.com.br, marciosoaresms@globo.com

(C)Associação de Medicina Intensiva Brasileira, 2007 mas, resultam em maiores satisfação e percepção da qualidade da assistência prestada ao paciente na UTI. Unitermos: comunicação, espiritualidade, ética, família, terminalidade da vida, terapia intensiva

\section{SUMMARY}

BACKGROUND AND OBJECTIVES: Caring for the families is one of the major tasks of the global care of patients admitted to the intensive care unit (ICU). In the context of a terminally ill patient or a patient in whom the recovery from the acute illness is unlikely, dealing with and caring for their family members becomes even more important as the patient will not be awake in most of situations. Family members have specific needs and present with high incidence of symptoms of stress, depression, anxiety and related disorders during the ICU of their beloved one, which can even persist late after the patient's death.

CONTENTS: Review of selected studies on the care of family members of patients at the end-of-life admitted to the ICU published at the PubMed database during the last 20 years.

CONCLUSIONS: Recent literature is plenty of evidence that strategies directed to care of family members, such as improvement of the communication process, prevention of conflicts, and spiritual care, can improve satisfaction and perception of quality on the care of patients at the end-of-life in the ICU.

Key Words: communication end-of-life, ethics family, intensive care, spirituality.

\section{INTRODUÇÃO}

O cuidado dos familiares é uma das partes mais importantes do cuidado global dos pacientes internados nas unidades de terapia intensiva (UTI). No contexto de um paciente terminal ou no qual as perspectivas de recuperação são muito improváveis, esta face do cuidado assume uma importância ainda maior, pois na maioria 
das vezes o paciente não estará desperto e será preciso lidar e cuidar dos seus familiares. Os familiares têm necessidades específicas e apresentam freqüências elevadas de estresse, distúrbios do humor e ansiedade durante o acompanhamento da internação na UTI, e que muitas vezes persistem após a morte do seu ente querido ${ }^{1,2}$. Atualmente, muitos profissionais de saúde das UTI ainda acreditam que o cuidado dos familiares dependa basicamente de habilidades e características individuais, e que esta responsabilidade seja exclusiva de profissionais específicos como psicólogos e assistentes sociais. Entretanto, embora esses profissionais tenham extrema importância nesse processo, o cuidado dos familiares é de responsabilidade de todos na UTI. É também lamentável que o cuidado da família seja tão pouco (ou não seja) abordado na maioria dos cursos de graduação da área da saúde. Por exemplo, programas educacionais voltados para preparar estudantes para lidarem com familiares de pacientes terminais têm resultados bastante satisfatórios ${ }^{3}$. Contudo, o momento atual é também extremamente oportuno para a sua discussão com o destaque que lhe é devido. A literatura recente está repleta de evidências de que estratégias voltadas para os familiares como a melhoria da comunicação, da prevenção de conflitos e do conforto espiritual, para citar algumas, resultam em maiores satisfação e percepção da qualidade da assistência prestada ao paciente na UTI. Neste artigo, os termos "família" e "familiares" são utilizados de modo amplo, englobando não apenas os parentes consangüíneos e cônjuges, mas todos os que fazem parte do círculo afetivo do paciente.

Foram selecionados artigos sobre o cuidado de familiares de pacientes em final de vida publicados nos últimos 20 anos na PubMed (www.pubmed.gov). Utilizaram-se unitermos: end-of-life, family care, communication, intensive care unit, palliative care.

\section{COMPREENDENDO AS NECESSIDADES DOS FA- MILIARES DE PACIENTES}

Nas situações de terminalidade, familiares de pacientes têm necessidades específicas que têm sido avaliadas em diversos estudos. Estas necessidades foram sumarizadas nas recomendações da Society of Critical Care Medicine para as situações de terminalidade na UTI e são as seguintes 4 .

- Estar próximo ao paciente;

- Sentir-se útil para o paciente;

- Ter ciência das modificações do quadro clínico;
- Compreender o que está sendo feito no cuidado e porque;

- Ter garantias do controle do sofrimento e da dor;

- Estar seguro de que a decisão quanto a limitação do tratamento curativo foi apropriada;

- Poder expressar os seus sentimentos e angústias;

- Ser confortado e consolado;

- Encontrar um significado para a morte do paciente.

Atender às necessidades dos familiares é uma das responsabilidades da equipe da UTI. A avaliação cuidadosa destas necessidades fornece subsídios importantes para a melhoria da compreensão, da satisfação e da capacidade para participar nas decisões relacionadas ao cuidado de alguém, que na maioria das vezes não poderá decidir por si próprio.

\section{PREPARAÇÃO DO AMBIENTE DA UTI PARA OS FAMILIARES DE PACIENTES EM SITUAÇÕES TERMINAIS}

As UTI sempre foram percebidas pela população leiga e mesmo por alguns profissionais de saúde de outras especialidades como lugares de muito sofrimento, frios e pouco acolhedores. A filosofia da humanização das UTI representou um grande avanço para a mudança desta percepção. Entretanto, este processo de "humanização" ainda é bastante tímido, pois a estrutura física e organizacional da maioria das UTI ainda prioriza a conveniência dos profissionais de saúde, deixando as prioridades de pacientes e seus familiares em segundo plano. Poucas são as UTI que dispõem de boxes individualizados, locais reservados para a comunicação com familiares e políticas de flexibilização do acesso ou mesmo de acesso livre aos familiares de pacientes, mesmo daqueles em situação de terminalidade. Estas necessidades são identificadas de modo recorrente dentre os principais fatores associados com a ocorrência de estresse, ansiedade e depressão nos familiares, bem como pela insatisfação com as assistências prestadas ao paciente ${ }^{1,2,5}$. Dentre as questões ambientais, o nível de ruído dentro de muitas UTI beira o insuportável para os profissionais de saúde, pacientes e familiares. Níveis elevados de ruídos constantes estão relacionados com burnout em profissionais de saúde ${ }^{6}$ são imediatamente apontados pelos familiares como indicadores de um ambiente desacolhedor na UTI.

$\mathrm{O}$ argumento de que a estrutura física de uma UTI foi concebida dentro de um padrão desejável em uma época passada, não pode servir de pretexto para o imobilismo no paradigma do cuidado a ser prestado aos 
familiares. Medidas organizacionais como o controle dos níveis de ruído, existência de um local (por mais elementar que seja) reservado e de um grupo limitado de pessoas para a informação dos familiares os quais possam ser identificados como responsáveis pelo cuidado do paciente, e a mobilização da equipe para uma política mais liberal dos horários de acesso dos familiares de pacientes em situação de terminalidade não demandam investimentos financeiros ou mudanças estruturais de grande porte, mas fundamentalmente de uma mudança cultural dos membros da equipe da UTI. Na flexibilização das políticas de visitação, a autorização da entrada mais de um familiar ao mesmo tempo é desejável. Para aqueles que experimentam a perda de um ente querido, este esforço organizacional pode representar muito. Wall e col. relataram que a satisfação de familiares com a assistência prestada na UTI aos pacientes que faleceram foi maior do a que de familiares dos pacientes que receberam alta ${ }^{7}$.

\section{COMUNICAÇÃO DURANTE CONFERÊNCIAS E ENCONTROS COM FAMILIARES SOBRE AS SITUA- ÇÕES DE TERMINALIDADE}

O processo de comunicação é a pedra angular do cuidado dos familiares. Um das tarefas mais importantes de um intensivista é fornecer, de modo apropriado, informações claras e realistas, mas também compassivas e solidárias. Particularmente, nas situações de terminalidade, os encontros podem ser difíceis e complexos quando não existe uma relação previamente estabelecida entre intensivistas e familiares. No estudo FAMIREA, realizado em 43 UTI francesas, $73 \%$ dos familiares e $84 \%$ dos cônjuges dos pacientes apresentavam sintomas de estresse, ansiedade ou depressão. Quando ajustados para características relacionadas ao paciente e dos próprios familiares, os principais fatores associados com a presença destes sintomas foram a ausência de encontros regulares com os médicos e de um local próprio para estes encontros, e principalmente a percepção de contradições nas informações recebidas $^{1}$. Cerca de metade dos familiares relataram que as informações sobre os pacientes foram passadas de forma inapropriada, e que a compreensão pelos familiares foi relacionada com tempo despendido durante os encontros ${ }^{5}$. Os sintomas de estresse, ansiedade e depressão podem perdurar muito tempo após a morte do paciente ${ }^{2}$.

Cicely Saunders, fundadora dos cuidados paliativos, observou que "o modo pelo qual as pessoas morrem permanece na memória dos que continuam vivos"6. No caso de familiares vivenciando uma situação de terminalidade, esta observação pode ser ao mesmo tempo um risco ou uma oportunidade. Uma boa comunicação depende fundamentalmente da capacidade de ouvir. É preciso haver atenção para não desperdiçar as oportunidades durante os encontros com os familiares e nas discussões sobre terminalidade e cuidados de final de vida $^{9-11}$. A comunicação deve ser feita de modo claro e com a utilização de termos que sejam compreensíveis pelos familiares, pois a utilização de expressões técnicas ou do uso do jargão médico pode impedir que o significado e a intenção de algumas assertivas não sejam assimilados adequadamente, o que pode gerar conflitos e sensações de "abandono" no cuidado do paciente e dos próprios familiares ${ }^{9-11}$.

A duração dos encontros é também de extrema importância, e encontros de duração reduzida estão relacionados à insatisfação com a assistência ${ }^{12}$. A relação contrária, isto é tempos maiores associados com uma maior satisfação, já foi igualmente documentada ${ }^{13}$. O tempo despendido em um número excessivo de procedimentos e tarefas que um paciente grave deman$\mathrm{da}$, compete e acaba muitas vezes por limitar o tempo dispensado à sua família. Entretanto, a equipe da UTI deve se organizar para diminuir este desequilíbrio no processo de cuidado de pacientes e familiares.

Uma medida simples e para a qual já existem evidências para o seu uso rotineiro é o fornecimento de uma brochura ou folheto informativo sobre o processo de cuidado, definição de alguns termos técnicos e de intervenções, e da estruturação física e organizacional da UTI ${ }^{14}$. Recentemente, o benefício do fornecimento de uma brochura específica para familiares de pacientes internados em UTI cuja expectativa era o óbito foi testada em um ensaio clínico ${ }^{15}$. No texto inicial desta brochura, além da descrição das informações que poderiam ser encontradas no documento, havia uma afirmação explícita de solidariedade da equipe da UTI. Os familiares que receberam a brochura além dos cuidados habituais prestados nas UTI apresentaram ao final de três meses do óbito do paciente menor incidência de sintomas de estresse pós-traumático, ansiedade e depressão e menor necessidade de fármacos psicoativos. Embora o tempo despendido aos familiares no primeiro encontro tenha sido maior no grupo da intervenção, o fornecimento da brochura reduziu de modo significativo a necessidade de informações complementares nos encontros subseqüentes. 


\section{CUIDADO ESPIRITUAL}

A espiritualidade é uma característica humana que, dentre outros aspectos, possibilita ao indivíduo encontrar significado e propósito para a sua vida ${ }^{16}$. Embora estejam relacionadas, espiritualidade e religião não são equivalentes. As situações que antecedem e envolvem os processos de morte e morrer estão entre aquelas em que a espiritualidade e a necessidade de conforto espiritual são mais evidentes. A despeito disso, poucos são os estudos disponíveis sobre o tema em familiares e pacientes internados em UTI. Em estudo realizado por Wall e col. ${ }^{17}$, com familiares de pacientes em situação de terminalidade na UTI, o grau de satisfação com o cuidado geral dos pacientes foram altamente correlacionados à satisfação com o cuidado espiritual. Dentre os aspectos avaliados, a disponibilidade ou a presença de um religioso (do hospital ou trazido pelo familiar) durante a visita na UTI foi o principal fator apontado. A visão de muitos intensivistas de que os cuidados médico e espiritual não sejam complementares é no mínimo insensível nas situações de terminalidade. Citando Robert Nelson: "A nossa (dos intensivistas) habilidade de atender as necessidades espirituais e religiosas na UTI não significa a fusão das funções de um representante religioso com as de um médico. $\mathrm{Na}$ verdade, ela requer uma capacidade de reflexão e atenção dos nossos próprios valores, sentimentos e crenças sobre como nós nos esforçamos para encontrar algum significado para o sofrimento que presenciamos"18.

\section{CONCLUSÃO}

Cuidar dos familiares dos pacientes sempre foi antes de tudo um ato de solidariedade e dependente das habilidades e do conhecimento tácito de cada indivíduo, mas é também hoje um conjunto de estratégias e intervenções testadas e fundamentadas cientificamente que devem fazer parte do processo de formação de todo profissional de saúde da UTI.

\section{REFERÊNCIAS}

01. Pochard F, Azoulay E, Chevret S et al - Symptoms of anxiety and depression in family members of intensive care unit patients: ethical hypothesis regarding decision-making capacity. Crit Care Med, 2001;29:1893-1897.

02. Abbott KH, Sago JG, Breen CM et al - Families looking back: one year after discussion of withdrawal or withholding of life-sustaining support. Crit Care Med, 2001;29:197-201.

03. Fineberg IC - Preparing professionals for family conferences in palliative care: evaluation results of an interdisciplinary approach. J Palliat Med, 2005;8:857-866.

04. Truog RD, Cist AF, Brackett SE et al - Recommendations for end-of-life care in the intensive care unit: The Ethics Committee of the Society of Critical Care Medicine. Crit Care Med, 2001;29:2332-2348.

05. Azoulay E, Pochard F, Chevret S et al - Half the family members of intensive care unit patients do not want to share in the decision-making process: a study in 78 French intensive care units. Crit Care Med, 2004;32:1832-1838.

06. Donchin $\mathrm{Y}$, Seagull FJ - The hostile environment of the intensive care unit. Curr Opin Crit Care, 2002;8:316-320.

07. Wall RJ, Curtis JR, Cooke CR et al - Family satisfaction in the ICU: differences between families of survivors and nonsurvivors. Chest 2007;132:1425-1433.

08. Saunders C - Pain and Impending Death, em: Wall PD, Melzack R - Textbook of Pain. New York, Churchill Livingstone, 1989;621-624.

09. Stapleton RD, Engelberg RA, Wenrich MD et al - Clinician statements and family satisfaction with family conferences in the intensive care unit. Crit Care Med, 2006;34:1679-1685.

10. West HF, Engelberg RA, Wenrich MD et al - Expressions of nonabandonment during the intensive care unit family conference. J Palliat Med, 2005;8:797-807.

11. Curtis JR, Engelberg RA, Wenrich MD et al - Missed opportunities during family conferences about end-of-life care in the intensive care unit. Am J Respir Crit Care Med, 2005;171:844-849.

12. Azoulay E, Pochard F, Chevret S et al - Meeting the needs of intensive care unit patient families: a multicenter study. Am J Respir Crit Care Med, 2001;163:135-139.

13. McDonagh JR, Elliott TB, Engelberg RA et al - Family satisfaction with family conferences about end-of-life care in the intensive care unit: increased proportion of family speech is associated with increased satisfaction. Crit Care Med, 2004;32:1484-1488.

14. Azoulay E, Pochard F, Chevret S et al - Impact of a family information leaflet on effectiveness of information provided to family members of intensive care unit patients: a multicenter, prospective, randomized, controlled trial. Am J Respir Crit Care Med, 2002;165:438-442.

15. Lautrette A, Darmon M, Megarbane B et al - A communication strategy and brochure for relatives of patients dying in the ICU. $\mathrm{N}$ Engl $\mathrm{J}$ Med, 2007;356:469-478.

16. Dyson J, Cobb M, Forman D - The meaning of spirituality: a literature review. J Adv Nurs, 1997;26:1183-1188.

17. Wall RJ, Engelberg RA, Gries CJ et al - Spiritual care of families in the intensive care unit. Crit Care Med, 2007;35:1084-1090.

18. Nelson RM - The compassionate clinician: attending to the spiritual needs of self and others. Crit Care Med, 2005;33:2841-2842. 